\title{
COMPARACIÓN DE MEDIDAS ZOOMÉTRICAS EN LAS GALLINAS BALEARES
}

\author{
COMPARISON OF ZOOMETRIC MEASUREMENTS IN BALEARIC HENS
}

\author{
Méndez, $Y .^{1 *}$, Pons, $A .^{2}$ y Francesch, $A{ }^{3}$ \\ ${ }^{1}$ Patronat per a la Recuperació i Defensa de les Races Autòctones de les Illes Balears (PRAIB). Oficina \\ Escorxador Insular. Eivissa. España. *yommenn@hotmail.com \\ ${ }^{2}$ Departament de Producció Animal. Unitat de Recursos Genètics Animals. Institut de Biologia Animal deles \\ Illes Balears S.A. (IBABSA). Sineu. España. \\ ${ }^{3}$ IRTA. Centre Mas de Bover. Costantí. España.
}

\section{Palabras clave adicionales}

Medidas corporales. Medidas craneales. Índices corporales. Aves de corral.

\section{RESUMEN}

Se han tomado un total de 26 medidas craneales y corporales y 8 índices en 42 gallinas baleares de cada una de las razas Ibicenca, Menorquina y Mallorquina bajo condiciones de manejo estándar. La gallina lbicenca ha sido la más pesada, con el cráneo y la quilla más largos y con mayor diámetro del tarso. La Menorquina ha mostrado los ojos más alargados, la cresta y las orejillas más grandes y las barbillas más largas y también han sido mayores el cuello, dorso, cola, ala plegada, muslo, medida ornitológica y envergadura. La gallina Mallorquina ha presentado menor peso e inferiores valores de las mediciones. En cuanto a los índices, la gallina lbicenca ha obtenido un mayor índice craneal e índice de orejillas; la Menorquina mayor índice de barbillas y la Mallorquina superior índice de cresta. La Mallorquina presentó el índice de pechuga más bajo.

\section{SUMMARY}

A total of 26 head and body measurements and 8 indexes have been taken in 42 hens of each Balearian breed: Ibizan, Minorca and Majorcan. These hens have been bred in standard conditions. The results have shown that Ibizan has been the heaviest one, and the one which has scored the longest cranium and keel of sternum too. Furthermore it has also reported a higher tarsus

Presentado al Congreso SERGA (2010, Asturias).

\section{AdDiTIONAL KEYWORDS}

Body measurements. Head measurements. Body indexes. Poultry.

diameter. Minorca has achieved the highest eye and wattle length and the biggest comb and ear lobes, the longest neck, back, tail, folding wing and thigh and the biggest ornithological measure and wingspan. Majorcan is the lightest one and it has scored minor values. Ibizan hen has obtained a higher cranium and ear lobe index. Minorca has scored the higher value for the wattle index and Majorcan has achieved a bigger comb index. Majorcan hen showed the lower chest index.

\section{INTRODUCCIÓN}

Las medidas corporales de las aves se han estudiado para diferentes fines. En algunos trabajos se ha estudiado la relación de algunas medidas zoométricas con el peso corporal, por ejemplo, en pollos indígenas africanos (Guèye et al., 1998). También se han usado medidas zoométricas para estudiar el dimorfismo sexual (Pérez et al., 2004; Badubi et al., 2006).

Aunque no hay muchos trabajos al respecto, también pueden usarse para comparar razas de una misma especie, recientemente, se ha descrito por Francesch et al. (2010) una metodología para caracterizar morfológicamente razas de gallinas y se ha mostrado su aplicación en la comparación 


\section{MÉNDEZ, PONSYFRANCESCH}

Tabla I. Ls medias \pm EE de las medidas corporales tomadas en gallinas de las razas baleares: Ibicenca (IB), Menorquina (ME) y Mallorquina (MA). (Ls means \pm SE of body measurements taken in hens of each Balearic breed: Ibizan (IB), Minorca (ME) and Majorcan (MA)).

\begin{tabular}{lccc}
\hline Medida & IB & ME & MA \\
\hline Peso & $2442,33 \pm 49,71^{\mathrm{a}}$ & $2071,47 \pm 48,38^{\mathrm{b}}$ & $1763,93 \pm 46,02^{\mathrm{c}}$ \\
Medición ornitológica & $570,20 \pm 5,00^{\mathrm{b}}$ & $616,55 \pm 4,80^{\mathrm{a}}$ & $526,83 \pm 4,56^{\mathrm{c}}$ \\
Envergadura & $779,05 \pm 4,09^{\mathrm{b}}$ & $814,08 \pm 3,99^{\mathrm{a}}$ & $746,43 \pm 3,79^{\mathrm{c}}$ \\
Longitud cuello & $141,66 \pm 3,52^{\mathrm{c}}$ & $174,21 \pm 3,43^{\mathrm{a}}$ & $154,78 \pm 3,26^{\mathrm{b}}$ \\
Longitud dorso & $213,33 \pm 3,86^{\mathrm{b}}$ & $231,60 \pm 3,76^{\mathrm{a}}$ & $196,95 \pm 3,57^{\mathrm{c}}$ \\
Longitud quilla & $126,16 \pm 1,87^{\mathrm{a}}$ & $118,00 \pm 1,82^{\mathrm{b}}$ & $108,81 \pm 1,73^{\mathrm{c}}$ \\
Ángulo de pecho & $75,08 \pm 1,55^{\mathrm{a}}$ & $74,68, \pm 1,51^{\mathrm{a}}$ & $72,28 \pm 1,44^{\mathrm{a}}$ \\
Longitud cola & $161,86 \pm 3,34^{\mathrm{b}}$ & $182,37 \pm 3,21^{\mathrm{a}}$ & $144,88 \pm 3,05^{\mathrm{c}}$ \\
Longitud ala plegada & $212,83 \pm 1,34^{\mathrm{b}}$ & $229,66 \pm 1,31^{\mathrm{a}}$ & $205,64 \pm 1,24^{\mathrm{c}}$ \\
Longitud muslo & $143,88 \pm 1,32^{\mathrm{b}}$ & $149,42 \pm 1,29^{\mathrm{a}}$ & $129,12 \pm 1,23^{\mathrm{c}}$ \\
longitud tarso & $101,11 \pm 0,89^{\mathrm{a}}$ & $103,34 \pm 0,87^{\mathrm{a}}$ & $89,62 \pm 0,83^{\mathrm{b}}$ \\
Diámetro tarso a-p & $14,55 \pm 0,16^{\mathrm{a}}$ & $13,37 \pm 0,16^{\mathrm{b}}$ & $12,50 \pm 0,15^{\mathrm{c}}$ \\
Diámetro tarso I-m & $10,96 \pm 0,13^{\mathrm{a}}$ & $9,79 \pm 0,12^{\mathrm{b}}$ & $9,36 \pm 0,12^{\mathrm{b}}$ \\
Longitud dedo medio & $59,71 \pm 0,58^{\mathrm{a}}$ & $58,05 \pm 0,56^{\mathrm{a}}$ & $52,74 \pm 0,54^{\mathrm{b}}$ \\
\hline
\end{tabular}

Las medias con letra diferente en una misma fila son significativamente diferentes $(p \leq 0,05)$.

de las razas Penedesenca (Francesch y Jordà, 1988) y Empordanesa (Francesch, 1994).

Este trabajo es una adaptación y aplicación de dicho método para comparar las tres gallinas autóctonas baleares.

\section{MATERIAL Y MÉTODOS}

Se han realizado mediciones en 42 gallinas de 52 semanas de vida de cada una de las razas baleares: Ibicenca, Menorquina y Mallorquina según el método de caracterización morfológica propuesto y descrito por Francesch et al. (2010), al que se ha añadido la medida del diámetro del tarso antero posterior, que se obtiene de medir la parte media del hueso metatarso en dirección cráneo-caudal sin hacer presión sobre la piel de recubrimiento y tres índices corporales, el del tarso, el del pico y el pectoral. El índice de pico se ha obtenido dividiendo la longitud del pico por la anchura de éste y el índice pectoral o de la pechuga, dividiendo la longitud de la quilla por el ángulo de la pechuga. El índice de tarso, se ha calculado como el cociente entre el diámetro antero posterior y el latero medial del tarso.

Se han tomado un total de 26 medidas zoométricas, 12 craneales y 14 corporales, que se muestran en las las tablas I y II, usando como instrumentos de peso y medición: balanza electrónica $(1 \mathrm{~g})$, cinta métrica $( \pm 1 \mathrm{~mm})$, pie de rey $( \pm 1 \mathrm{~mm})$ y un goniómetro.

Las 26 medidas zoométricas y los índices se han tratado estadísticamente como variables dependientes, estudiándose el factor raza, con tres niveles (gallina Ibicenca IB, gallina Menorquina ME y gallina Mallorquina MA). El modelo se ha resuelto mediante el General Linear Model (GLM) del SAS 9.1. Se ha aceptado un nivel de significación de 0,05 .

\section{RESULTADOSYDISCUSIÓN}

De las 14 medidas corporales estudiadas (tabla I), se han encontrado diferencias en todas menos en el ángulo de pecho. La gallina Ibicenca ha resultado ser la más pesada. La gallina Menorquina ha presentado valores superiores para la medición ornitológica y envergadura además de lon- 


\section{COMPARACIÓN DE MEDIDASZOOMÉTRICAS EN LAS GALLINASBALEARES}

Tabla II. Ls medias $\pm \mathrm{EE}$ de las medidas craneales tomadas en gallinas de las razas baleares: Ibicenca (IB), Menorquina (ME) y Mallorquina (MA). (Ls means \pm SE of head measurements taken in hens of each Balearic breed: Ibizan (IB), Minorca (ME) and Majorcan (MA)).

\begin{tabular}{lccc}
\hline Medida & IB & ME & MA \\
\hline Longitud cráneo & $46,80 \pm 0,74^{\mathrm{a}}$ & $40,45 \pm 0,72^{\mathrm{b}}$ & $39,83 \pm 0,68^{\mathrm{b}}$ \\
Anchura cráneo & $32,16 \pm 0,28^{\mathrm{a}}$ & $31,74 \pm 0,27^{\mathrm{b}}$ & $30,86 \pm 0,26^{\mathrm{b}}$ \\
Longitud cresta & $62,16 \pm 2,06^{\mathrm{b}}$ & $75,55 \pm 2,01^{\mathrm{a}}$ & $51,66 \pm 1,91^{\mathrm{c}}$ \\
Anchura cresta & $31,36 \pm 1,61^{\mathrm{b}}$ & $45,31 \pm 1,57^{\mathrm{a}}$ & $20,90 \pm 1,49^{\mathrm{c}}$ \\
Longitud ocular & $10,88 \pm 0,16^{\mathrm{b}}$ & $11,81 \pm 0,16^{\mathrm{a}}$ & $10,78 \pm 0,15^{\mathrm{b}}$ \\
Anchura ocular & $5,86 \pm 0,15^{\mathrm{a}}$ & $6,10 \pm 0,14^{\mathrm{a}}$ & $5,74 \pm 0,14^{\mathrm{a}}$ \\
Longitud pico & $27,64 \pm 0,35^{\mathrm{a}}$ & $26,95 \pm 0,34^{\mathrm{a}}$ & $26,16 \pm 0,32^{\mathrm{a}}$ \\
Anchura pico & $14,22 \pm 0,22^{\mathrm{a}}$ & $13,71 \pm 0,22^{\mathrm{a}}$ & $12,90 \pm 0,21^{\mathrm{b}}$ \\
Longitud orejillas & $24,88 \pm 0,84^{\mathrm{b}}$ & $39,08 \pm 0,85^{\mathrm{a}}$ & $22,76 \pm 0,77^{\mathrm{b}}$ \\
Anchura orejillas & $9,83 \pm 0,56^{\mathrm{b}}$ & $25,24 \pm 0,54^{\mathrm{a}}$ & $11,45 \pm 0,52^{\mathrm{b}}$ \\
Longitud barbillas & $26,22 \pm 1,01^{\mathrm{b}}$ & $36,58 \pm 1,06^{\mathrm{a}}$ & $22,48 \pm 1,01^{\mathrm{b}}$ \\
Anchura barbillas & $21,69 \pm 0,73^{\mathrm{a}}$ & $24,08 \pm 0,71^{\mathrm{a}}$ & $18,24 \pm 0,68^{\mathrm{b}}$ \\
\hline
\end{tabular}

Las medias con letra diferente en una misma fila son significativamente diferentes $(p \leq 0,05)$.

gitud del cuello, dorso y cola. La Ibicenca ha mostrado el valor más elevado para longitud de la quilla y la Menorquina la mayor longitud del ala plegada y del muslo.

Si se hace referencia a la longitud del tarso y del dedo medio, las gallinas Ibicenca y Menorquina han mostrado valores más elevados que la Mallorquina. Sin embargo, al considerar el diámetro del tarso, la gallina Ibicenca es la que ha obtenido mayores valores en las dos mediciones.

La gallina Mallorquina ha presentado valores inferiores en las medidas corporales salvo en la longitud del cuello, por encima de la Ibicenca, pero sin superar a la Menorquina.

Las medidas corporales de la gallina Menorquina indican mayor estilización, lo cual podría responder a su selección como ave ornamental.

Tabla III. Ls medias \pm EE de los índices corporales tomados en gallinas de las razas baleares: Ibicenca (IB), Menorquina (ME) y Mallorquina (MA). (Ls means \pm SE of body indexes taken in hens of each Balearic breed: Ibizan (IB), Minorca (ME) and Majorcan (MA)).

\begin{tabular}{lccc}
\hline Índices corporales & IB & $M E$ & $M A$ \\
\hline Índice craneal & $1,46 \pm 0,02$ a & $1,27 \pm 0,02^{\mathrm{b}}$ & $1,29 \pm 0,02^{\mathrm{b}}$ \\
Índice ocular & $1,94 \pm 0,05^{\mathrm{a}}$ & $1,88 \pm 0,05^{\mathrm{a}}$ & $1,96 \pm 0,05^{\mathrm{a}}$ \\
Índice cresta & $2,07 \pm 0,08^{\mathrm{b}}$ & $1,73 \pm 0,08^{\mathrm{c}}$ & $2,64 \pm 0,08^{\mathrm{a}}$ \\
Índice orejillas & $2,62 \pm 0,09^{\mathrm{a}}$ & $1,56 \pm 0,09^{\mathrm{c}}$ & $2,15 \pm 0,08^{\mathrm{b}}$ \\
Índice barbillas & $1,24 \pm 0,05^{\mathrm{b}}$ & $1,53 \pm 0,05^{\mathrm{a}}$ & $1,26 \pm 0,04^{\mathrm{b}}$ \\
Índice pico & $1,96 \pm 0,03^{\mathrm{a}}$ & $1,98 \pm 0,03^{\mathrm{a}}$ & $2,04 \pm 0,03^{\mathrm{a}}$ \\
Índice pechuga & $1,69 \pm 0,02^{\mathrm{a}}$ & $1,74 \pm 0,03^{\mathrm{a}}$ & $1,51 \pm 0,03^{\mathrm{b}}$ \\
Índice tarso & $1,33 \pm 0,02^{\mathrm{a}}$ & $1,37 \pm 0,02^{\mathrm{a}}$ & $1,34 \pm 0,02^{\mathrm{a}}$ \\
\hline
\end{tabular}

Las medias con letra diferente en una misma fila son significativamente diferentes $(p \leq 0,05)$. 


\section{MÉNDEZ, PONSYFRANCESCH}

De las 12 medidas craneales estudiadas (tabla II), se han presentado diferencias en todas menos la anchura ocular y la longitud del pico. La gallina Ibicenca ha mostrado mayor longitud de cráneo que las otras. En la Menorquina son mayores la longitud y anchura de la cresta y orejillas y longitud ocular y de las barbillas. La anchura de sus barbillas presenta valores similares a la Ibicenca, pero superiores a la Mallorquina.

De los 8 índices corporales, no se observan diferencias significativas en el índice ocular, el de pico y el del tarso (tabla III).

La gallina Ibicenca ha mostrado un cráneo de apariencia más alargada y orejillas menos redondeadas que el resto, pues posee un mayor índice craneal y de orejillas. La gallina Mallorquina, seguida de la Ibicenca, ha presentado el mayor valor para el índice de la cresta, y la Menorquina el menor, lo que sería debido a su gran longitud y anchura de cresta, con lo cual el índice es menor y la cresta, por tanto, resulta más grande. Pero la Menorquina es la que ha presentado un mayor índice de barbillas, lo cual se traduce en que las barbillas de la Mallorquina e Ibicenca resultan más redondeadas.

El índice de la pechuga es similar para la Ibicenca y Menorquina, por ello la aparien-

\section{BIBLIOGRAFÍA}

Badubi, S.S., Rakereng, M. and Marumo, M. 2006. Morphological characteristics and feed resources available for indigenous chickens in Botswana. Livest. Res. Rural Dev., 18 (1). http://www.Irrd.org/lrrd18/1/badul18003.htm. (16/03/10).

Francesch, A. 1994. La raza de gallinas Empordanesa. Arte avícola, 2: 15-18.

Francesch, A. y Jordà, A. 1988. La raza de gallinas del Penedés: una labor de hoy que conecta con el pasado. Selecciones Avícolas XXX, 10: 307-314.

Francesch, A., Villalba, I. and Cartañá, M. 2010. Methodology for morphological characterization cia de su pechuga es más alargada, de manera que la Mallorquina con quilla más corta y ángulo de pechuga similar, ha presentado el menor índice y por tanto, una pechuga de apariencia más redondeada.

Conectando con el trabajo de Francesch et al. (2010), se pueden hacer algunas comparaciones a grandes rasgos con las razas catalanas, Pendesenca y Empordanesa que, a pesar de sus diferencias, tienen mucha semejanza morfológica, y comparando los resultados con las baleares, parece que la Mallorquina entraría en el grupo formado por las dos catalanas mientras que la Ibicenca se apartaría por ser más pesada y la Menorquina, por ser de estructuras más alargadas, a la vez que con un mayor desarrollo de la cresta, barbillas y orejillas.

La gallina Mallorquina presenta resultados similares a los de Pérez et al. (2004) que estudiaron medidas zoométricas similares a las de este trabajo en gallinas de Villa Clara (Cuba).

\section{AGRADECIMIENTOS}

A las Asociaciones de Criadores de Gallinas Mallorquina, Menorquina e Ibicenca.

of chicken and its application to compare Penedesenca and Empordanesa breeds. Anim. Genet. Res. Info. (in press).

Guèye, E.F., Ndiaye, A. and Branckaert, R.D.S. 1998. Prediction of body weight on the basis of body measurements in mature indigenous chickens in Senegal. Livest. Res. Rural Dev., 10 (3). http://.cipav.org.co//rrd//rrd10/3/scene103. htm. (19/04/10).

Pérez, A., Polanco, G. and Pérez, Y. 2004. Morphological characteristics of local chicken ecotypes in Villa Clara Province in Central Cuba. Livest. Res. Rural Dev., 16 (10). http://wwwIrrd.org/lrrd16/10/pere16076.htm. (16/03/10). 